TITLE:

\title{
A Novel Tenorrhaphy Suture Technique with Tissue Engineered Collagen Graft to Repair Large Tendon Defects
}

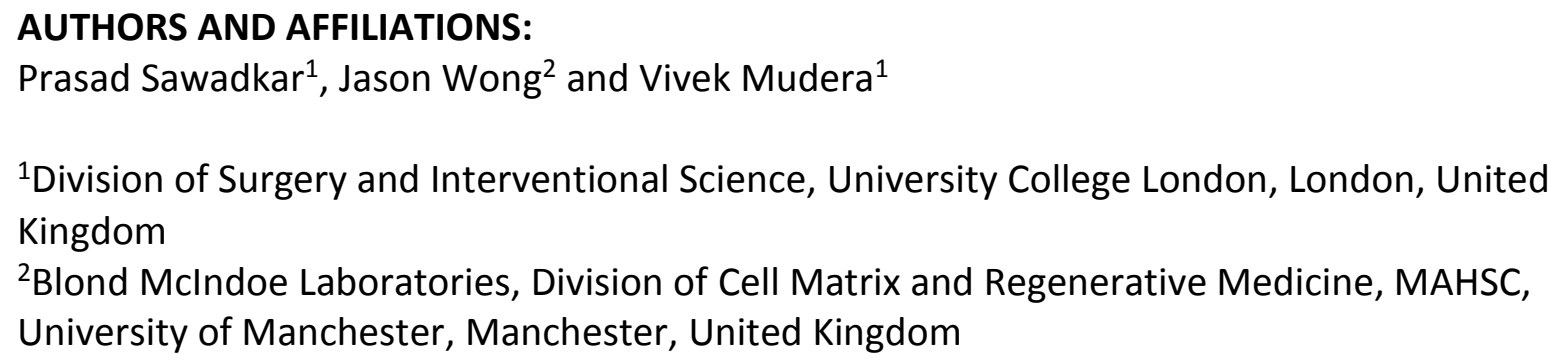

\section{KEYWORDS}

Tendon repair, tissue engineering, collagen, tendon graft, suture technique, tendon

\section{SUMMARY}

In this paper, we present an in vitro and in situ protocol to repair a tendon gap of up to $1.5 \mathrm{~cm}$ by filling it with engineered collagen graft. This was performed by developing a modified suture technique to take the mechanical load until the graft matures into the host tissue.

\section{ABSTRACT}

Surgical management of large tendon defects with tendon grafts is challenging, as there are a finite number of sites where donors can be readily identified and used. Currently, this gap is filled with tendon auto-, allo-, xeno-, or artificial grafts, but clinical methods to secure them are not necessarily translatable to animals because of the scale. In order to evaluate new biomaterials or study a tendon graft made up of collagen type 1, we have developed a modified suture technique to help maintain the engineered tendon in alignment with the tendon ends. Mechanical properties of these grafts are inferior to the native tendon. To incorporate engineered tendon into clinically relevant models of loaded repair, a strategy was adopted to offload the tissue engineered tendon graft and allow for the maturation and integration of the engineered tendon in vivo until a mechanically sound neo-tendon was formed. We describe this technique using incorporation of the collagen type 1 tissue engineered tendon construct.

\section{INTRODUCTION}

43 Tendon rupture may occur due to extrinsic factors such as traumatic lacerations or excessive loading of the tendon. Due to the external tensile forces placed on a tendon repair, a gap 
inevitably forms with most tendon repair techniques. Currently, tendon defects/gaps are filled with auto-, allo-, xeno- or artificial grafts, but their availability is finite, and the donor site is a source of morbidity.

The tissue-engineered approach to fabricate tendon graft from a natural polymer such as collagen has the distinctive advantage of being biocompatible and can provide vital extracellular matrix (ECM) components that facilitate cell integration. However, due to a lack of fibrillar alignment, the mechanical properties of the engineered tendon (ET) are inferior to the native tendon. To increase mechanical properties of the weaker collagen, many methods have been used, such as physical cross-linking under vacuum, UV radiation, and dehydrothermal treatments ${ }^{1}$. Also, through chemical cross-linking with riboflavin, enzymatic and non-enzymatic methods increased collagen density and the Young's modulus of the collagen in vitro ${ }^{2,3}$. However, by adding cross-linking agents, biocompatibility of the collagen is compromised, as studies have shown a $33 \%$ alteration in mechanical properties and $40 \%$ loss of cell viability ${ }^{3-5}$. Gradual accruement of alignment and mechanical strength can be obtained through cyclic loading ; however, this can be efficiently acquired in vivo ${ }^{7}$.

For ET to integrate in vivo and acquire strength without the need for chemical alteration, one approach would be to use a stabilizing suture technique to hold the weaker construct in place. Most tendon repairs rely on the suture design to hold tendon ends together; hence modification of these existing techniques could provide a logical solution ${ }^{8,9}$.

Until the 1980s, 2-strand repairs were widely used, but recent surgical literature describes the use of 4 strands, 6 strands or even 8 strands in repair ${ }^{10,11}$. In 1985, Savage described 6-strand suture techniques with 6 anchor points, and it was significantly stronger than the Bunnell suture technique that uses 4 strands ${ }^{12}$. Also, 8 -strand repairs are $43 \%$ stronger than other strands in cadaver and in situ models, but these repairs are not widely practiced as it becomes technically difficult to reproduce the repairs accurately ${ }^{13-16}$. Therefore, a greater number of core suture strands relates to a proportional increase in biomechanical properties of the repaired tendon. However, there is a loss of cell viability around the suture points, and trauma from excessive suturing can be to the detriment of the tendon, which can compromise tendon healing ${ }^{17}$. Suture techniques should provide a strong geometric repair that is balanced and relatively inelastic to minimize tendon gapping after repair. In addition, the location of the suture and its knots have to be strategically placed in order for them not to interfere with gliding, blood supply and healing until accruement of adequate strength has been obtained ${ }^{10,18}$.

81

To establish feasibility to secure weaker ET graft or other graft material in between ruptured tendon, we have developed a novel suture technique that can offload the graft so that it can mature and gradually integrate into the host tissue in vivo.

\section{PROTOCOL:}

86

87

Note: Experiment design and ethical approval were obtained from UCL Institutional Review Board (IRB). All experiments were carried out as per regulation of Home Office and guidelines of Animals 
(scientific procedure) Act 1986 with revised legislation of European Directive 2010/63/EU (2013). Rabbits were inspected by a named veterinary surgeon (NVS) periodically and twice in a day by a named animal care and welfare officer (NACWO) (As per guidelines and regulations of Home office). They did not show any sign of pain until they were euthanized.

\section{Preparation of Tissue Engineered Tendon (ET) Graft}

1.1. To fabricate the collagen hydrogel, add $4 \mathrm{~mL}$ of rat tail collagen type 1 monomeric collagen solution $(2.15 \mathrm{mg} / \mathrm{mL}$ in $0.6 \%$ acetic acid with $0.2 \% \mathrm{w} / \mathrm{v}$ of total protein) and $500 \mu \mathrm{L}$ of $10 \mathrm{x}$ Minimal Essential Medium. Neutralize this by titrating against $5 \mathrm{M}$ and $1 \mathrm{M}$ sodium hydroxide and add $500 \mu \mathrm{L}$ of Dulbecco's Modified Eagle Medium (DMEM).

1.2. Pour $5 \mathrm{~mL}$ of this solution into a custom built rectangular metal mold ( $33 \mathrm{~mm} \times 22 \mathrm{~mm} \times 10$ $\mathrm{mm}, 120 \mathrm{~g}$ weight) (Figure 1). Keep the mold in a $\mathrm{CO}_{2}$ incubator at $37{ }^{\circ} \mathrm{C}$ and $5 \% \mathrm{CO}_{2}$ for 15 minutes to allow matrix assembly ${ }^{19}$.

\section{Fabrication of the Graft}

2.1. After polymerization, remove the collagen hydrogel from the mold and place in a standard plastic compression assembly (Figure $\mathbf{2 A})^{19}$.

2.2. Place the collagen hydrogel in between two $50 \mu \mathrm{m}$ nylon mesh sheets and apply a static load of $120 \mathrm{~g}$ (total surface area $7.4 \mathrm{~cm}^{2}$, which is a pressure equivalent to $1.6 \mathrm{kPa}$ ) for 5 minutes to remove interstitial fluid from the hydrogel (Figure 2A). Use four layers of filter paper to absorb the discharged fluid from hydrogels.

2.3. Use four layers of compressed gels rolled on top of each other (Figure 2B) and cut into 15 $\mathrm{mm}$ segments (Figure $\mathbf{2 C}$ ) to fabricate the ET.

Note: New Zeland white male rabbits of age 16 - 25 weeks were used in the experiments.

2.4. Sedate animals with an intramuscular (i.m.) dose of Hypnorm $(0.3 \mathrm{mg} / \mathrm{mL})$ and euthanize by administering an overdose of pentobarbitone.

2.5. Immediately after euthanasia, trim the hair on both hind legs. Then with a size 20 surgical blade, make a $9 \mathrm{~cm}$ incision around the inferior tibiofibular area to expose the tibialis posterior (TP) tendon.

2.6. With the same sized surgical blade, excise lapine TP tendons with an average length of 70 $\mathrm{mm}$ and keep moist in PBS during the experimental process to avoid drying.

\section{Developed Novel Tenorrhaphy Technique}


Note: The sutures (see Table of Materials) are non-absorbable and made from an isotactic crystalline stereoisomer of polypropylene, which is a synthetic linear polyolefin. The core interlocking sutures were mainly consisting of 3-0 and the peripheral sutures were 6-0. These were the two main sutures used in all experiments.

3.1. With a surgical blade, cut the TP tendon at the midpoint. Excise a $15 \mathrm{~mm}$ segment of the tendon from the middle of the tendon and replace it with the ET collagen graft (Figure 2D). Interlock the 3-0 suture proximally away from native tendon ends (Figure 3A).

3.2. Pass the 3-0 core sutures above the entire length of the graft and interlock distally away from the cut end.

3.3. Secure both ends of the ET to the native tendon with 6-0 and continuous running sutures around the periphery by coupling two tendon ends (Figure 3B). This is done so that the graft can be moved easily on the suture by placing tension on the native tendon ${ }^{20}$.

3.4. After securing the suture as described above, manually ensure that the tension on the sutures is appropriate and that there is no flaccidity in the entirety of the suture.

\section{REPRESENTATIVE RESULTS}

We have used collagen grafts fabricated from type I collagen, as this is the predominant protein found in the tendon. It constitutes almost $95 \%$ of total collagen in the tendon; hence, collagen has exhibited all ideal properties for mimicking tendon in vivo ${ }^{21,22}$.

In this study, the type I collagen used was extracted from rat tail tendon and dissolved in the acetic acid $(2.15 \mathrm{mg} / \mathrm{mL})$. To polymerize this collagen, it was neutralized with sodium hydroxide in vitro, which formed non-cross-linked anisotropic collagen fibrils. This hydrogel contains $98 \%$ fluid and could mimic living tissue in vivo within 20 minutes during fabrication ${ }^{23}$. However, this hydrogel is mechanically weak; therefore, to increase mechanical properties, we have developed a method for rapid compression of collagen hydrogel by a technique known as 'plastic compression', where the degree of compression is directly proportional to the applied weight on the top and released fluid from the fluid leaving surface (FLS) ${ }^{19}$.

Spiral rolling of this graft increases its mechanical properties ${ }^{19}$, but the graft remains significantly weaker than the native tendon. To address this issue, we have developed a novel modified suture technique by placing suture points, not at the edge of ruptured tendons but proximally and distally away. Thus, the strength of the repair is on the sutures and suture points and not on the mechanically weaker tendon graft.

To demonstrate the functionality of the developed novel suture technique, a lapine TP tendon was excised. The gap was filled with a $15 \mathrm{~mm}$ long tendon graft secured with 6-0 sutures, and 30 interlocked sutures were placed at $70 \mathrm{~mm}$ to act as load barriers (Figure 3A). The mean break strength of repair was $50.62 \pm 8.17 \mathrm{~N}$, which was significantly higher $(p<0.05)$ than that of the control Kessler repair of $12.49 \pm 1.62 \mathrm{~N}$ (Figure 4A). Hence, core suture length and their 
interlocking away from the tendon ends significantly influence resistance of the tendon and the repairs from failing at higher magnitude forces ${ }^{24,25}$.

This resistance was inadequate in the control repairs which caused early repair failure and strain failure of more than $20 \%$ on the tendon. However, this is a physiological anomaly, as tendons in vivo are never subject to $20 \%$ strain due to there not being enough space for a tendon to extend that much; therefore to test feasibility of the suture technique in vivo models, we have performed repair in situ and calculated a mean break strength of $24.60 \pm 3.92 \mathrm{~N}$, which is significantly higher than the control mean break strength of $13.98 \pm 2.26 \mathrm{~N}$ (Figure 4B).

\section{FIGURES AND TABLE LEGENDS}

Figure 1: Neutralized collagen hydrogel (pH 7.4) (pink color) cast in the stainless steel mold. Gel was allowed to remain in a $\mathrm{CO}_{2}$ incubator at $37^{\circ} \mathrm{C}$ for 20 minutes for fibrillogenesis to occur. The scale bar is shown at the bottom.

Figure 2: Plastic compression process. (A) The collagen hydrogel placed in between nylon meshes with a constant static load of 120 grams applied. Drained fluid was absorbed by four layers of filter paper. The arrow shows the fluid leaving surface (FLS) for the gel. (B) Four layers of compressed collagen sheets were rolled along the axis to form 'engineered tendon' (ET). (C) The section of ET was cut into $15 \mathrm{~mm}$ segments to mimic tendon. (D) The tendon defect was created in the native tendon (NT) by excising a $15 \mathrm{~mm}$ segment of the posterior tibial tendon, and the defect was filled with ET. This panel was modified from previous work ${ }^{26}$.

Figure 3: (A) Tendon defect was filled with ET and secured with 6-0 sutures, and the 3-0 interlocking four strand suture technique was performed passing above graft in the $30 \mathrm{~mm}$ region. Block arrow shows the starting point for the suture and the blank arrow shows the end point of the suture. This panel was modified from previous work ${ }^{26}$. (B) Feasibility of performing developed suture technique in a space inside lapine model (in situ).

Figure 4: Mechanical strength. (A) A mechanical test output of the repair and (B) in situ mechanical test output (Error bars $=\mathrm{SD} ;{ }^{*} p<0.05$, one-way ANOVA with Bonferroni correction). This panel was modified from previous work ${ }^{26}$.

\section{DISCUSSION}

In this study, tissue engineered type I collagen grafts was chosen as a tendon graft because collagen is a natural polymer and used as a biomaterial for various tissue engineering applications ${ }^{27,28}$. Also, tendon collagen constitutes $60 \%$ of the dry mass of tendon, out of which $95 \%$ is type 1 collagen ${ }^{21,29-32}$. For successful engraftment to occur, mechanical properties of the graft should ideally match the native tendon ${ }^{33}$; however, with current engineering techniques, the mechanical properties of ET $(4.41 \mathrm{~N})$ are significantly inferior to the native tendon (NT) $(261.08 \mathrm{~N})^{33}$. It is proposed that this is due to the highly organized hierarchical arrangement of collagen fibril in the native tendon, which remains a challenge to engineer and match its mechanical properties ${ }^{34}$. We have tried to increase the density of the ET matrix by applying a 
static weight of compression to the collagen hydrogel ${ }^{33}$; however, the architectural complexity from which the tendon acquires its strength is more intricate. Methods to accrue mechanical strength arguably are best attained in vivo, where the host biological processes can act on the remodelling of the extracellular matrix. Therefore, in this study, another strategy was adopted to modify the current suture technique as post tendon repair; the mechanical strength of the repaired tendon graft is entirely dependent on the suture technique ${ }^{8,9}$. Hence, by modifying existing suture techniques, we can offload the engineered tendon graft until cell and ECM induced remodelling occurs as a new approach.

To date, there are various suture techniques available to repair the tendon, none of which is a gold standard; however, the modified Kessler suture technique is widely used to repair tendons because it is less obstructive and damaging to tendons ${ }^{35,36}$. The flexor digitorum profundus muscle tendon of lambs, when sutured with the 6-strand Savage technique, was reported to have a break strength of $51.3 \mathrm{~N}$, but when a modified Kessler suture technique was used, the break strength was $69.0 \mathrm{~N}^{7}$. However, in this study, when the tendon gap of $15 \mathrm{~mm}$ was filled with ET and repaired with Modified Kessler suture technique, the repair failed at an early stage with a break strength of $12.49 \mathrm{~N}$ (Figure 4). This low value makes the technique clinically irrelevant. Similar findings have been reported by De Wit et al. in a porcine flexor repair tendon model, suggesting that Kessler repair failed at suture rupture by reducing gapping by $15 \%$ as compared to cruciate repair, where gapping is reduced by $87 \%$ and repair failed at suture pull-out ${ }^{38}$. Thus, there is a need for another strong suture technique, which could hold mechanically weaker ET in place.

A novel modified suture technique was developed by using four core sutures over the entire length of the ET and above the opposite tendon. These sutures were interlocked onto the suture material itself at some distance away from each tendon end. This is mainly because it has been reported that putting suture knots at equal distance and equal load sharing tension on all suture strands increases their mechanical properties ${ }^{39}$. A balanced repair can also be achieved by keeping a continuous suture, and staggering the repair to allow for compression at the repair site $^{40}$.

In this study, 3-0 sutures were used for outer interlocked sutures considering that rabbit TP tendon has a length, width and thickness of $62.4 \mathrm{~mm}, 5 \mathrm{~mm}$ and $1.5 \mathrm{~mm}$, respectively. $6-0$ sutures were used to hold the ET in place. Although we have tried other absorbable suture materials, it would not be appropriate as they become weaker over a period in vivo ${ }^{41}$. A primary reason polypropylene sutures was selected is because they are a monofilament as well as nonabsorbable and they do not cause structural or tensional modifications under load ${ }^{42}$. We tested all sutures from 2-0 to 7-0, but 3-0 and 6-0 were found to be ideal candidates for our experiments 26.

The primary reason for using 4 strand repair was to avoid excessive damage to ruptured tendon ends with a greater number of suture strands as it has been reported that a normal surgical suture in a tendon results in the formation of an acellular region ${ }^{43}$. It has been hypothesized that this is due to the cells migrating out from the compressive load that is put on the tendon, and 
normally these cells are subject to tensile loading ${ }^{17}$. This migration of cells away from the suture could then cause weakening of the matrix as there is a paucity of cells to maintain and turnover the matrix, which could cause early tendon failure ${ }^{17}$. We can use more strands of sutures that are biomechanically twice as strong (ex vivo) than 4 -strand sutures ${ }^{11,12,44,45}$; however, these repairs are not widely practiced and their clinical limitations are currently being evaluated ${ }^{13-16}$.

The placement of the suture knot is important but there are arguments for and against externalizing the suture. Having the suture on the outer surface can potentially snag against structures like tendon pulleys and reduce glide. In a study, the areas where suture knots are placed inside illustrated a decrease in gliding resistance compared with the Kessler repair, which has suture knots outside ${ }^{46}$. Studies conducted in the canine model concluded that at a higher magnitude of the force, fewer suture knots located outside the repair and away from the tendon ends had survived compared with those located inside the repair ${ }^{47,48}$. However, internalizing the knot potentially reduces the contact surface of the healing tendon. There is also the consideration that tissue damage arises from the suture needle piercing the tendon and the greater number of passes relates to the increased tendon trauma ${ }^{49}$.

To secure ET in between the tendon gap, a standard of running sutures ${ }^{50}$ along the edge of the tendon and ET was performed. This was done because there was a need for peripheral sutures that are strong enough to hold the ET in place in the initial phase of healing until cell and ECM induced remodelling could occur ${ }^{50}$. The major problem was the variation in the mechanical properties of the NT and ET, which could result in early gap formation although the ET was stress shielded. On the other hand, applying a more secure technique such as horizontal mattress intrafiber sutures ${ }^{51}$, Halsted continuous horizontal mattress sutures ${ }^{52,53}$, cross stitch epitendinous repair techniques ${ }^{54-57}$ or running lock sutures ${ }^{58,59}$ would have ruptured ET as it is fragile. Thus, we chose running sutures as a peripheral suture technique which is simple and holds the ET intact in all directions.

From a tissue engineering perspective, we need to study whether this method can be used to fill a tendon gap greater than $1.5 \mathrm{~cm}$. To use this graft in human clinical trials, we need to further investigate the immunological response to the xenogeneic source of collagen although this can be achieved by developing clinical grade collagen. The protocol described herein establishes the feasibility of the developed suture technique within available anatomical spaces in a porcine lapine model. This developed suture technique has suture points proximally and distally equidistance away from ruptured tendon ends so that engineered tendon graft could be off loaded. Hence, it could mature and integrate in vivo.

\section{ACKNOWLEDGEMENTS:}

The authors would like to acknowledge UCL for funding this project.

\section{DISCLOSURES:}

The authors declare that they have no conflicts of interest.

\section{REFERENCES:}


1 Wollensak, G., Spoerl, E. \& Seiler, T. Riboflavin/ultraviolet-a-induced collagen crosslinking for the treatment of keratoconus. American Journal of Ophthalmology 135, 620-627 (2003).

2 Tanzer, M. L. Cross-Linking of Collagen. Science 180, 561-566 (1973).

3 Reiser, K., McCormick, R. J. \& Rucker, R. B. Enzymatic and nonenzymatic cross-linking of collagen and elastin. FASEB Journal 6, 2439-2449 (1992).

4 Kanungo, B. P. \& Gibson, L. J. Density-property relationships in collagenglycosaminoglycan scaffolds. Acta Biomaterialia 6, 344-353 (2010).

5 Weadock, K. S., Miller, E. J., Bellincampi, L. D., Zawadsky, J. P. \& Dunn, M. G. Physical crosslinking of collagen fibers: comparison of ultraviolet irradiation and dehydrothermal treatment. Journal of Biomedical Materials Research 29, 1373-1379 (1995).

6 Kalson, N. S. et al. Slow Stretching That Mimics Embryonic Growth Rate Stimulates Structural and Mechanical Development of Tendon-Like Tissue In Vitro. Developmental Dynamics 240, 2520-2528 (2011).

7 Torigoe, K. et al. Mechanisms of collagen fibril alignment in tendon injury: from tendon regeneration to artificial tendon. Journal of Orthopaedic Research 29, 1944-1950 (2011).

8 Ketchum, L. D. Suture materials and suture techniques used in tendon repair. Hand Clinics 1, 43-53 (1985).

9 Lawrence, T. M. \& Davis, T. R. A biomechanical analysis of suture materials and their influence on a four-strand flexor tendon repair. The Journal of Hand Surgery 30, 836-841 (2005).

10 Strickland, J. W. Development of flexor tendon surgery: Twenty-five years of progress. The Journal of Hand Surgery 25, 214-235 (2000).

11 Moriya, K. et al. Clinical outcomes of early active mobilization following flexor tendon repair using the six-strand technique: short- and long-term evaluations. The Journal of Hand Surgery, European Volume (2014).

12 Savage, R. In vitro studies of a new method of flexor tendon repair. Journal of Hand Surgery 10, 135-141 (1985).

13 Uslu, M. et al. Flexor tendons repair: effect of core sutures caliber with increased number of suture strands and peripheral sutures. A sheep model. Orthopaedics \& Traumatology: Surgery \& Research : OTSR 100, 611-616 (2014).

14 Osei, D. A. et al. The Effect of Suture Caliber and Number of Core Suture Strands on Zone II Flexor Tendon Repair: A Study in Human Cadavers. Journal of Hand Surgery 39, 262-268 (2013).

15 Dovan, T. T., Ditsios, K. T. \& Boyer, M. I. Eight-strand core suture technique for repair of intrasynovial flexor tendon lacerations. Techniques in Hand \& Upper Extremity Surgery 7, 70-74 (2003).

16 Silva, M. J. et al. The effects of multiple-strand suture techniques on the tensile properties of repair of the flexor digitorum profundus tendon to bone. The Journal of Bone and Joint surgery. American Volume 80, 1507-1514 (1998).

17 Wong, J. K., Alyouha, S., Kadler, K. E., Ferguson, M. W. \& McGrouther, D. A. The cell biology of suturing tendons. Matrix Biology 29, 525-536 (2010).

18 Strickland, J. W. Flexor Tendon Injuries: II. Operative Technique. The Journal of the American Academy of Orthopaedic Surgeons 3, 55-62 (1995). 
19 Brown, R. A., Wiseman, M., Chuo, C. B., Cheema, U. \& Nazhat, S. N. Ultrarapid Engineering of Biomimetic Materials and Tissues: Fabrication of Nano- and Microstructures by Plastic Compression. Advanced Functional Materials 15, 1762-1770 (2005). Sawadkar, P., Alexander, S. \& Mudera, V. Tissue-engineered collagen grafts to treat large tendon defects. Regenerative Medicine 9, 249-251 (2014).

21 Evans, J. H. \& Barbenel, J. C. Structural and mechanical properties of tendon related to function. Equine veterinary journal 7, 1-8 (1975).

22 Riley, G. P. et al. Glycosaminoglycans of human rotator cuff tendons: changes with age and in chronic rotator cuff tendinitis. Annals of the Rheumatic Diseases 53, 367-376 (1994).

23 Bell, E., Ivarsson, B. \& Merrill, C. Production of a tissue-like structure by contraction of collagen lattices by human fibroblasts of different proliferative potential in vitro. Proceedings of the National Academy of Sciences of the United States of America 76, 1274-1278 (1979).

24 Kim, H. M. et al. Technical and biological modifications for enhanced flexor tendon repair. The Journal of Hand Surgery 35, 1031-1038 (2010).

25 Kim, J. B., de Wit, T., Hovius, S. E., McGrouther, D. A. \& Walbeehm, E. T. What is the significance of tendon suture purchase? The Journal of Hand Surgery, European Volume 34, 497-502 (2009).

26 Sawadkar, P. et al. Development of a surgically optimized graft insertion suture technique to accommodate a tissue-engineered tendon in vivo. BioResearch Open Access 2, 327-335 (2013).

27 Hadjipanayi, E. et al. Mechanisms of structure generation during plastic compression of nanofibrillar collagen hydrogel scaffolds: towards engineering of collagen. Journal of Tissue Engineering and Regenerative Medicine 5, 505-519 (2011).

28 Micol, L. A. et al. High-density collagen gel tubes as a matrix for primary human bladder smooth muscle cells. Biomaterials 32, 1543-1548 (2011).

29 Lian Cen , W. L., Lei Cui, Wenjie Zhang, and Yilin Cao. Collagen Tissue Engineering: Development of Novel Biomaterials and applications. Pediatric Research 63, 492-496 (2008).

30 Harris, M. T. et al. Mesenchymal stem cells used for rabbit tendon repair can form ectopic bone and express alkaline phosphatase activity in constructs. Journal of Orthopaedic Research 22, 998-1003 (2004).

31 Butler, D. L. et al. The use of mesenchymal stem cells in collagen-based scaffolds for tissue-engineered repair of tendons. Nature Protocols 5, 849-863 (2010).

32 Cen, L., Liu, W., Cui, L., Zhang, W. \& Cao, Y. Collagen Tissue Engineering: Development of Novel Biomaterials and Applications. Pediatric Research 63, 492-496 (2008).

33 Yamaguchi, H., Suenaga, N., Oizumi, N., Hosokawa, Y. \& Kanaya, F. Will Preoperative Atrophy and Fatty Degeneration of the Shoulder Muscles Improve after Rotator Cuff Repair in Patients with Massive Rotator Cuff Tears? Advances in Orthopedics 2012, 195876 (2012).

34 Silver, F. H., Freeman, J. W. \& Seehra, G. P. Collagen self-assembly and the development of tendon mechanical properties. Journal of Biomechanics 36, 1529-1553 (2003). 
35 Schneppendahl, J. et al. Initial stability of two different adhesives compared to suture repair for acute Achilles tendon rupture--a biomechanical evaluation. International Orthopaedics 36, 627-632 (2012).

36 Herbort, M. et al. Biomechanical comparison of the primary stability of suturing Achilles tendon rupture: a cadaver study of Bunnell and Kessler techniques under cyclic loading conditions. Archives of Orthopaedic and Trauma Surgery 128, 1273-1277 (2008).

37 Piskin, A. et al. [Tendon repair with the strengthened modified Kessler, modified Kessler, and Savage suture techniques: a biomechanical comparison]. Acta Orthopaedica et Traumatologica Turcica 41, 238-243 (2007).

38 de Wit, T., Walbeehm, E. T., Hovius, S. E. \& McGrouther, D. A. The mechanical interaction between three geometric types of nylon core suture and a running epitenon suture in repair of porcine flexor tendons. The Journal of Hand Surgery, European Volume 38, 788-794 (2013).

39 Trail, I. A., Powell, E. S. \& Noble, J. The mechanical strength of various suture techniques. Journal of Hand Surgery 17, 89-91 (1992).

40 Wong, J. K. \& Peck, F. Improving results of flexor tendon repair and rehabilitation. Plastic and Reconstructive Surgery 134, 913e-925e (2014).

41 Amis, A. A. Absorbable sutures in tendon repair. Journal of Hand Surgery 21, 286 (1996).

42 Faggioni, R. \& de Courten, C. [Short and long-term advantages and disadvantages of prolene monofilament sutures in penetrating keratoplasty]. Klinische Monatsblatter fur Augenheilkunde 200, 395-397 (1992).

43 Wong, J. K., Cerovac, S., Ferguson, M. W. \& McGrouther, D. A. The cellular effect of a single interrupted suture on tendon. Journal of Hand Surgery 31, 358-367 (2006).

44 Savage, R. \& Risitano, G. Flexor tendon repair using a "six strand" method of repair and early active mobilisation. Journal of Hand Surgery 14, 396-399 (1989).

45 Okubo, H., Kusano, N., Kinjo, M. \& Kanaya, F. Influence of different length of core suture purchase among suture row on the strength of 6-strand tendon repairs. Hand Surgery 20, 19-24 (2015).

46 Noguchi, M., Seiler, J. G., 3rd, Gelberman, R. H., Sofranko, R. A. \& Woo, S. L. In vitro biomechanical analysis of suture methods for flexor tendon repair. Journal of Orthopaedic Research 11, 603-611 (1993).

47 Aoki, M., Pruitt, D. L., Kubota, H. \& Manske, P. R. Effect of suture knots on tensile strength of repaired canine flexor tendons. Journal of Hand Surgery 20, 72-75 (1995).

48 Pruitt, D. L., Aoki, M. \& Manske, P. R. Effect of suture knot location on tensile strength after flexor tendon repair. The Journal of Hand Surgery 21, 969-973 (1996).

49 Khor, W. S. et al. Improving Outcomes in Tendon Repair: A Critical Look at the Evidence for Flexor Tendon Repair and Rehabilitation. Plastic and Reconstructive Surgery 138, 1045e-1058e (2016).

50 Strickland, J. W. Flexor Tendon Injuries: I. Foundations of Treatment. The Journal of the American Academy of Orthopaedic Surgeons 3, 44-54 (1995).

51 Mashadi, Z. B. \& Amis, A. A. Strength of the suture in the epitenon and within the tendon fibres: development of stronger peripheral suture technique. Journal of Hand Surgery 17, 172-175 (1992). 
43952 Wade, P. J., Muir, I. F. \& Hutcheon, L. L. Primary flexor tendon repair: the mechanical

440 limitations of the modified Kessler technique. Journal of Hand Surgery 11, 71-76 (1986).

$44153 \quad$ Wade, P. J., Wetherell, R. G. \& Amis, A. A. Flexor tendon repair: significant gain in strength from the Halsted peripheral suture technique. Journal of Hand Surgery 14, 232235 (1989).

54 Silfverskiold, K. L. \& May, E. J. Gap formation after flexor tendon repair in zone II. Results with a new controlled motion programme. Scandinavian Journal of Plastic and Reconstructive Surgery and Hand Surgery / Nordisk Plastikkirurgisk forening [and] Nordisk Klubb for Handkirurgi 27, 263-268 (1993). Silfverskiold, K. L., May, E. J. \& Tornvall, A. H. Gap formation during controlled motion after flexor tendon repair in zone II: a prospective clinical study. The Journal of Hand Surgery 17, 539-546 (1992). suture for flexor tendon repair in dogs. The Journal of Hand Surgery 13, 553-558 (1988). Surgery 20, 261-266 (1995). 